\title{
PRODUÇÃO CIENTÍFICA DOS CONGRESSOS BRASILEIROS DE ENFERMAGEM NOS ANOS 70: CONTRIBUIÇÃO PARA A ENFERMAGEM PEDIÁTRICA
}

\author{
SCIENTIFIC PRODUCTION FROM THE BRAZILIAN NURSING CONGRESSES IN THE 70'S: CONTRIBUITION \\ FOR PEDIATRIC NURSING \\ PRODUCCIÓN CIENTÍFICA DE LOS CONGRESSOS BRASILEÑOS DE ENFERMERIA, EN LOS ANOS 70: \\ CONTRIBUCIÓN PARA LA ENFERMERÍA DE PEDIÁTRICA
}

\section{Renata Oliveira da Costa ${ }^{1}$, Isabel Cristina dos Santos Oliveira ${ }^{2}$}

\begin{abstract}
RESUMO: Trata-se de um estudo histórico, que enfoca a produção científica acerca da temática "Saúde da Criança" dos Congressos Brasileiros de Enfermagem nos anos 70, destacando os temas oficiais e livres dos referidos eventos. Os objetivos são: verificar o número de temas oficiais e livres apresentados nos CBEn e identificar as temáticas relacionadas à saúde da criança. Constata-se que a referida temática foi pouco abordada, sendo que o XXVII CBEn, em 1975, e o XXX CBEn, em 1978, apresentaram os maiores percentuais, ou seja, 1,53\% de temas oficiais e $3,08 \%$ de temas livres relacionados à temática respectivamente.
\end{abstract}

PALAVRAS CHAVE: Enfermagem Pediátrica; Congressos; Saúde da Criança.

ABSTRACT: This is a historical study which focuses the scientific production on the "Child's Health" theme from Brazilian Nursing Congresses (CBEn) in the 70's, highlighting official and free themes from the referred events. The objectives of the study are: Verifying the number of official and free themes presented on the CBEn and Identifying subjects related to Child's Health. It was stated that the referred theme was not very mentioned, although the XXVI CBEn, in 1975, and the XXX CBEn in 1978 presented the greatest rates, $1,53 \%$ on official themes and $3,08 \%$ on free themes related to the subject described.

KEY WORDS: Pediatric Nursing; Congresses; Child Health.

RESUMEN: Estudio histórico, que enfoca la producción científica referente a la temática "salud del niño", de los Congresos Brasileños de Enfermería en los años 70, separando los temas oficiales y libres relacionados al evento. Los objetivos son: verificar el número de temas oficiales y libres presentados en los CBEn e identificar los temas relacionados con la salud del niño. Es evidente que el tema citado fue poco abordado, aún que el XXVII CBEn, en 1975, y el XXX CBEn, en 1978, habían presentado los mayores porcentajes, o sea, $1,53 \%$ de temas oficiales y $3,08 \%$ de temas libres relacionados con el tema respectivamente.

PALABRAS CLAVE: Enfermería Pediátrica; Congresos; Salud del Niño.
1 Graduanda do $8^{\circ}$ período do Curso de Graduação em Enfermagem e Obstetrícia da Escola de Enfermagem Anna Nery da Universidade Federal do Rio de Janeiro (EEAN/ UFRJ), RJ. Bolsista de Iniciação Científica / CNPq.

${ }^{2}$ Doutora em Enfermagem. Professora Adjunta do Departamento de Enfermagem Médico -Cirúrgica da EEAN/ UFRJ.Rio de Janeiro, RJ. Orientadora, Coordenadora do Projeto Integrado de Pesquisal CNPq. Líder do Grupo de Pesquisa - Saúde da Criançal Cenário Hospitalar. Pesquisadora/CNPq. E-mail: chabucris@ig.com.br 


\section{INTRODUÇÃO}

Desde 1947, quando ocorreu o $1^{\circ}$ Congresso Nacional de Enfermagem (atual - Congresso Brasileiro de Enfermagem) em São Paulo, nas dependências da Escola de Enfermagem da Universidade de São Paulo (USP), e por muito tempo, os CBEn representaram os únicos espaços de discussão, atualização e descrição dos trabalhos da enfermagem, pois permitiam envolver na discussão as enfermeiras de todo o Brasil. Ao término de cada congresso, eram aprovadas as recomendações que eram encaminhadas às diferentes instâncias políticas. Apesar da introdução de outros eventos mais setoriais ou de especialidades, os CBEn representam, até hoje, espaços privilegiados de encontro nacional das enfermeiras dos diferentes cenários de prática, em consonância com a sua finalidade, exposta desde 1947, pela Comissão de Estatuto da Associação Brasileira de Enfermagem ABEn. (CARVALHO, 1976).

Ainda, CARVALHO (1976, p. 9), ressalta que a" Associação realizará um ou mais Congressos anualmente para promover a aproximação das sócias, apresentação de trabalhos técnico-científicos e proporcionar aos seus membros facilidades para melhor conhecimento do País, em geral, e de desenvolvimento da enfermagem no Brasil, em particular."

Desde 1947 até a atualidade, os CBEn, refletem um movimento da enfermagem em busca de sua definição, enquanto profissão e uma prática social no conjunto das práticas da saúde, numa visão idealizada, normatizadora e descontextualizada, quando muito imitativa de outras realidades, para situar-se histórica e socialmente, acompanhando o movimento geral da construção do conhecimento. (BERTOLOZZI et al, 2000).

A dinâmica de apresentação dos temas dos CBEn possibilita entender as transformações histórico - sociais e econômicas do país, e principalmente, a trajetória histórica e a consolidação da profissão - enfermagem.

Considerando o discurso das enfermeiras nos Anais dos CBEn, acredito que possa entender uma das facetas para a institucionalização da especialidade - enfermagem pediátrica. Desta forma, o objeto de estudo é a produção científica dos Congressos Brasileiros de Enfermagem nos anos 70.

Este recorte temporal se justifica tendo em vista que, este estudo está inserido no Projeto Integrado de Pesquisa/CNPq, intitulado: "A Institucionalização da Enfermagem Pediátrica: da Prática à Especialidade nos anos 70", sendo que, em 1970, foi apresentado um tema livre no CBEn, que abordou a assistência integral ao prematuro, e em 1979 um tema livre, que enfocou a importância da assistência de enfermagem na realimentação do desnutrido em ambulatório.
Os objetivos do estudo são: verificar o número de temas oficiais e livres apresentados nos CBEn nos anos 70; e identificar as temáticas relacionadas à saúde da criança;

\section{AS TEMÁTICAS DOS CONGRESSOS BRASILEIROS DE ENFERMAGEM NOS ANOS 70: UMA SÍNTESE}

A década de 70 está relacionada diretamente a predominância da assistência curativa individualizada, favorecendo a expansão da rede de assistência médico-hospitalar privada. Os hospitais assumem uma posição central na prestação de saúde, acentuando cada vez mais a dicotomia entre as ações curativa e preventiva, e concentrando diversas especialidades médicas.

Vale destacar que para o aperfeiçoamento da assistência de enfermagem à criança, tornou-se necessário uma especialização em Enfermagem Pediátrica, que surgiu a partir da prática médica especializada (OLIVEIRA, 2003).

No que tange a enfermagem, nessa época houve um incremento dos cursos de pós-graduação com a criação de novos cursos de especialização, inclusive em Enfermagem Pediátrica. Segundo FERRIANI et al (1985), organiza-se curso de especialização, principalmente na área de pediatria, destacando-se o da Escola Paulista de Enfermagem (atual Departamento de Enfermagem da Universidade Federal de São Paulo), na década de 70. Após esta época, houve um crescimento progressivo da produção científica em enfermagem, determinada pelo aumento do número de cursos de pós-graduação lato sensu.

A implementação dos cursos de pós-graduação atende não só à demanda de enfermeiras qualificadas para o ensino, mas também a especialização crescente da medicina e da tecnologia hospitalar. Assim, os currículos de graduação e de pós-graduação em enfermagem concentram-se no enfoque curativo e nos estágios hospitalares. (BERTOLOZZI et al, 2000, p. 33).

No final da década de 70 ocorreu, no CBEn, uma aderência das temáticas à estrutural conjuntura da época, focalizando como tema central a situação de saúde no país e estimulando os profissionais a discutirem os desafios que se colocavam para a área.

Nessa década, observa-se uma oscilação dos temários dos CBEn das questões mais coletivas para assistência individual de enfermagem. Isso fica mais claro nos temas de cada congresso descritos sucintamente, a seguir. Em 1970, o XXII CBEn que ocorreu em São Paulo, teve como temas oficiais, a integração do recém - graduado na vida profissional, política da $A B E n$ e elementos básicos do diagnóstico (REBEn,1970).

No ano de 1971, no XXIII CBEn, Manaus, os temas oficiais foram, o princípio da investigação e o processo de observação sistematizada na 
enfermagem, o papel da enfermeira nos serviços de saúde diante da realidade regional ou local no Brasil, a participação da enfermeira no planejamento da saúde e recentes estudos e pesquisas nas especialidades de enfermagem (REBEn,1971). Em 1972, no XXIV CBEn, que ocorreu em Belo Horizonte, os temas oficiais foram a formação dos profissionais de enfermagem dos três níveis, o exercício profissional, participação da enfermeira no planejamento de saúde, e recentes pesquisas em enfermagem (CEBEn,1972). No ano de 1973, o XXV CBEn, realizado em João Pessoa, teve como temas oficiais, o currículo mínimo do curso de graduação em enfermagem e obstetrícia, recentes pesquisas em enfermagem, problemas relacionados com o ensino e o exercício da enfermagem nas áreas - médicocirúrgica, materno-infantil, administração, saúde pública e humanização da enfermagem (CEBEn,1973).

Em 1974, o XXVI CBEn realizado em Curitiba, teve como temas as necessidades psicossociais e psico-espirituais dos pacientes, liderança, recentes pesquisas em enfermagem, educação em enfermagem, aspectos assistenciais de enfermagem e problemas de infecções hospitalares (CEBEn,1974).

O XXVII CBEn que ocorreu no ano de 1975 em Salvador, teve como temas, integração do ensino e serviço de enfermagem, participação do pessoal nos programas de assistência à saúde nas populações da zona rural, problemas de assistências de enfermagem nos hospitais e clínicas particulares de grandes centros urbanos e recentes pesquisas em enfermagem (CEBEn,1975).. Em 1976, no XXVIII CBEn, que ocorreu no Rio de Janeiro teve como temas, a formação filosófica e religiosa da enfermeira, sistema de registro de enfermagem, projeção da ABEn na comunidade brasileira e pesquisa em enfermagem (CEBEn,1976)

No ano de 1977, o XXIX CBEn que ocorreu em Camboriú, teve como temas, situação de saúde no país - implicações para a enfermagem, relações da Enfermagem com as demais carreiras universitárias, a enfermeira e as habilitações em nível de $2^{\circ}$ grau, relação trabalho-aprendizagem segundo Piaget, e Licenciatura em Enfermagem (CEBEn,1977).

Em 1978, no XXX CBEn que ocorreu em Belém, teve como tema central o Sistema Nacional de Saúde, cujos temas oficiais foram: a enfermagem e o sistema de saúde, o ensino e a assistência de enfermagem diante dos programas de extensão e cobertura, as inovações no ensino superior de enfermagem diante da assistência de saúde à população - possibilidades e limitações, e as entidades de classe (ABEn, Conselho Federal de Enfermagem, Sindicato) ( CEBEn,1978). Já no ano de 1979, o XXXI CBEn que ocorreu em Fortaleza, teve como tema central: desafios da enfermagem brasileira, e os temas oficiais foram, a enfermagem e a estrutura social, reflexos sobre a prática profissional, e o preparo e aperfeiçoamento de recursos humanos para a enfermagem (CEBEn,1979).

\section{METODOLÓGIA}

Trata-se de um estudo histórico que, segundo CARDOSO (1994, p. 25) "Os estudos sóciohistoricamente determinados devem levar em consideração os fatos articulados de uma dada época".

As fontes primárias são os artigos publicados nos Anais dos CBEn (CEBEn, 1970, 1971, 1972, $1973,1974,1975,1976,1977,1978,1979$ ), existentes nos acervos das Bibliotecas a saber: Setorial de Pós- Graduação da Escola de Enfermagem Anna Nery da Universidade Federal do Rio de Janeiro (EEAN/UFRJ), Nacional, Faculdade de Enfermagem da Universidade do Estado do Rio de Janeiro (FENF/UERJ) e da Associação Brasileira de Enfermagem (ABEn - Seção Rio de Janeiro).

Os temas oficiais e livres dos CBEn de 1970 a 1976 foram publicados nos volumes da Revista Brasileira de Enfermagem (REBEn) dos respectivos anos. No entanto, é valido ressaltar que os referidos temas do XXII Congresso Brasileiro de Enfermagem, que ocorreu em São Paulo, em 1970, e o XXIII Congresso Brasileiro de Enfermagem, que ocorreu em Manaus, em 1971, foram publicados somente na Revista Brasileira de Enfermagem, não sendo publicados em Anais (REBEn,1970;1971).

As fontes secundárias são os livros que abordam os Congressos Brasileiros de Enfermagem e estudos referentes à enfermagem nas áreas de neonatologia e pediatria (teses, dissertações e periódicos nacionais).

De acordo com BRIGNOLI \& CARDOSO (1983), existem dois tipos de fontes históricas: primárias e secundárias. As fontes primárias correspondem aos documentos escritos publicados no período estudado ou antecedente e resultam do tema pesquisado, e as fontes secundárias são livros e/ ou artigos sobre o tema estudado, ou relacionado com ele.

Procedeu-se a análise estatística, com apresentação dos dados através de tabelas com freqüências absoluta e relativa, quadros e gráficos. $O$ tratamento estatístico "permite uma concentração maior e simplificação das informações, aproximandoas, facilitando desta forma as comparações, interpretações ou análise" (CERVO \& BERVIAN, 1996, p. 14).

\section{APRESENTAÇÃO E ANÁLISE DOS RESULTADOS}

$\mathrm{Na}$ análise dos dados referentes aos temas publicados nos Anais, foram encontrados um total de 197 temas oficiais e 227 temas livres. Na Tabela 1, verifica-se que no XXVI CBEn realizado em Curitiba, em 1974, foi apresentado $18,27 \%$ de temas oficiais e 
o XXX CBEn realizado em Belém, em 1978, teve 31,29\% de temas livres.

Tabela 1 - Total de temas oficiais e livres publicados nos Anais dos Congressos Brasileiros de Enfermagem nos anos 70

\begin{tabular}{|c|c|c|c|c|}
\hline \multirow{2}{*}{ ANAISI LOCALIANO } & \multicolumn{2}{|c|}{ TEMAS OFICIAIS } & \multicolumn{2}{|c|}{ TEMAS LIVRES } \\
\hline & $\mathbf{F}$ & $\%$ & $\mathbf{F}$ & $\%$ \\
\hline $\begin{array}{l}\text { XXII CBEn } \\
\text { São Paulo/SP - } 1970\end{array}$ & 6 & 3,05 & 28 & 12,33 \\
\hline $\begin{array}{l}\text { XXIII CBEn } \\
\text { Manaus/AM - } 1971\end{array}$ & 16 & 8,12 & 2 & 0,88 \\
\hline $\begin{array}{l}\text { XXIV CBEn } \\
\text { Belo Horizonte/MG - } 1972\end{array}$ & 23 & 11,68 & 19 & 8,37 \\
\hline $\begin{array}{l}\text { XXV CBEn } \\
\text { João Pessoa/PB - } 1973\end{array}$ & 31 & 15,74 & 7 & 3,08 \\
\hline $\begin{array}{l}\text { XXVI CBEn } \\
\text { Curitiba/PR - } 1974\end{array}$ & 36 & 18,27 & 27 & 11,89 \\
\hline $\begin{array}{l}\text { XXVII CBEn } \\
\text { Salvador/BA - } 1975\end{array}$ & 28 & 14,21 & 42 & 18,50 \\
\hline $\begin{array}{l}\text { XXVIII CBEn } \\
\text { Rio de Janeiro/RJ-1976 }\end{array}$ & 32 & 16,24 & 31 & 13,66 \\
\hline $\begin{array}{l}\text { XXIX CBEn } \\
\text { Camboriú(SC) - } 1977\end{array}$ & 9 & 4,57 & - & - \\
\hline $\begin{array}{l}\text { XXX CBEn } \\
\text { Belém(PA) - } 1978\end{array}$ & 4 & 2,03 & 71 & 31,29 \\
\hline $\begin{array}{l}\text { XXXI CBEn } \\
\text { Fortaleza(CE) - } 1979\end{array}$ & 12 & 6,09 & - & - \\
\hline TOTAL & 197 & 100 & 227 & 100 \\
\hline
\end{tabular}

Com base na análise dos Anais dos CBEn, constata-se que no XXIX CBEn realizado em Camboriú no ano de 1977, somente foram publicados os trabalhos que receberam prêmios, e o XXXI CBEn realizado em Fortaleza no ano de 1979, foram publicados nos Anais somente os trabalhos referentes aos temas oficiais.

Pode-se supor que o número de trabalhos apresentados no congresso aumenta no final da década de 70 , tendo em vista que nesta época já existiam cursos de pós-graduação estrito senso, em destaque, em 1972 o Curso de Mestrado da Escola de Enfermagem Anna Nery da Universidade Federal do Rio de Janeiro (EEAN / UFRJ).

$\mathrm{Na}$ Tabela 2, verifica-se que dos 197 temas oficiais, $10(5,07 \%)$ estão relacionados à saúde da criança, sendo que no XXVII CBEn realizado em Salvador, em 1975, foi apresentado 1,52\% de temas oficiais relacionados à temática.

Tabela 2 - Distribuição dos temas oficiais segundo à natureza

\begin{tabular}{|c|c|c|c|c|}
\hline \multirow[t]{2}{*}{ ANAISI LOCALI ANO } & \multicolumn{2}{|c|}{$\begin{array}{c}\text { TEMAS OFICIAIS } \\
\text { (Geral) }\end{array}$} & \multicolumn{2}{|c|}{$\begin{array}{l}\text { TEMAS OFICIAIS } \\
\text { (Saúde da Criança }\end{array}$} \\
\hline & $\mathbf{F}$ & $\%$ & $\mathbf{F}$ & $\%$ \\
\hline $\begin{array}{l}\text { XXII CBEn } \\
\text { São Paulo - } 1970\end{array}$ & 6 & 3,05 & - & - \\
\hline XXIII CBEn & 16 & 0,88 & 2 & 1,01 \\
\hline
\end{tabular}


Manaus - 1971

XXIV CBEn

Belo Horizonte -1972

XXV CBEn

João Pessoa - 1973

XXVI CBEn

Curitiba - 1974

XXVII CBEn

Salvador - 1975

XXVIII CBEn

Rio de Janeiro - 1976

XXIX CBEn

Camboriú - 1977

XXX CBEn

Belém - 1978

XXXI CBEn

Fortaleza - 1979

TOTAL

\begin{tabular}{|c|c|c|}
\hline 11,68 & 1 & 0,50 \\
\hline 15,74 & 2 & 1,01 \\
\hline 18,27 & 1 & 0,50 \\
\hline 14,21 & 3 & 1,52 \\
\hline 16,24 & 1 & 0,50 \\
\hline 4,57 & - & - \\
\hline 2,03 & - & - \\
\hline 6,09 & - & - \\
\hline 100 & 10 & 5,04 \\
\hline
\end{tabular}

$\mathrm{Na}$ Tabela 3, verifica-se que dos 227 temas livres, $17(7,48 \%)$ estão relacionados à saúde da em 1978, foram apresentados 3,08\% de temas livres criança, sendo que no XXX CBEn ocorrido em Belém,

relacionados à temática.

Tabela 3 - Distribuição dos temas livres segundo à natureza

\begin{tabular}{|c|c|c|c|c|}
\hline \multirow[t]{2}{*}{ ANAISI LOCALI ANO } & \multicolumn{2}{|c|}{$\begin{array}{c}\text { TEMAS OFICIAIS } \\
\text { (Geral) }\end{array}$} & \multicolumn{2}{|c|}{$\begin{array}{l}\text { TEMAS OFICIAIS } \\
\text { (Saúde da Criança }\end{array}$} \\
\hline & $\mathbf{F}$ & $\%$ & $\mathbf{F}$ & $\%$ \\
\hline $\begin{array}{l}\text { XXII CBEn } \\
\text { São Paulo - } 1970\end{array}$ & 28 & 12,33 & 3 & 1,32 \\
\hline $\begin{array}{l}\text { XXIII CBEn } \\
\text { Manaus - } 1971\end{array}$ & 2 & 0,88 & - & - \\
\hline $\begin{array}{l}\text { XXIV CBEn } \\
\text { Belo Horizonte - } 1972\end{array}$ & 19 & 8,37 & 1 & 0,44 \\
\hline $\begin{array}{l}\text { XXV CBEn } \\
\text { João Pessoa - } 1973\end{array}$ & 7 & 3,08 & - & - \\
\hline $\begin{array}{l}\text { XXVI CBEn } \\
\text { Curitiba - } 1974\end{array}$ & 27 & 11,89 & - & - \\
\hline $\begin{array}{l}\text { XXVII CBEn } \\
\text { Salvador - } 1975\end{array}$ & 42 & 18,50 & 1 & 0,44 \\
\hline $\begin{array}{l}\text { XXVIII CBEn } \\
\text { Rio de Janeiro - } 1976\end{array}$ & 31 & 13,66 & 5 & 2,20 \\
\hline XXIX CBEn & - & - & - & - \\
\hline
\end{tabular}




\begin{tabular}{l|c|c|c|c} 
Camboriú - 1977 & & & \\
$\begin{array}{l}\text { XXX CBEn } \\
\text { Belém - 1978 }\end{array}$ & 71 & 31,29 & 7 & 3,08 \\
$\begin{array}{l}\text { XXXI CBEn } \\
\text { Fortaleza }-1979\end{array}$ & - & - & - & - \\
\hline \multicolumn{1}{c|}{ TOTAL } & $\mathbf{2 2 7}$ & $\mathbf{1 0 0}$ & $\mathbf{1 7}$ & $\mathbf{7 , 4 8}$ \\
\hline
\end{tabular}

Observa-se que o maior percentual da produção científica, referente a temática - saúde da criança na sessão de temas livres foi no XXX CBEn, em 1978, que ocorreu em Belém; ano em que alguns cursos de pós-graduação já tinha sidos criados no Brasil.

Verifica-se que a pós-graduação traz vantagens na dinamização da pesquisa, que é imprescindível como processo de desenvolvimento para promoção do saber específico.
O Gráfico 1 ilustra o número reduzido de temas oficiais e livres referentes à temática - Saúde da Criança em relação outros temas abordados nos congressos no período de 1970 a 1979.

Gráfico 1 - Distribuição de temas oficiais e livres da área - saúde da criança e de natureza geral.

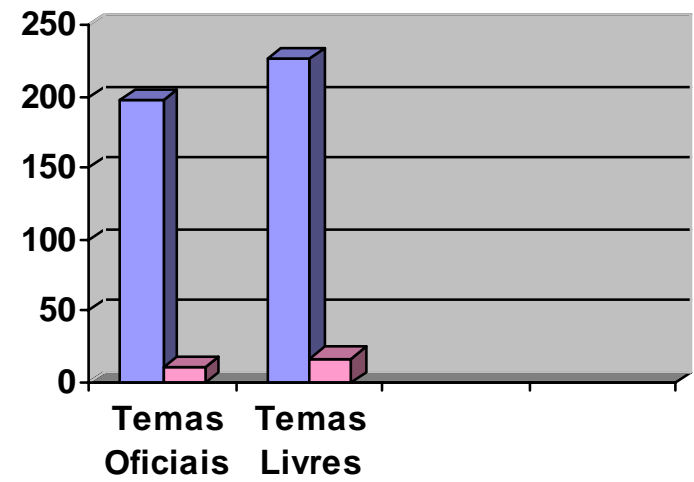

O Gráfico 02 ilustra um número reduzido de temas, sobre a saúde da criança, referentes ao meio extrahospitalar e uma predominância significativa do

\section{$\square$ geral \\ $\square$ saúde da criança}

Gráfico 02 - Distribuição dos temas oficiais e livres quanto à sua especificidade

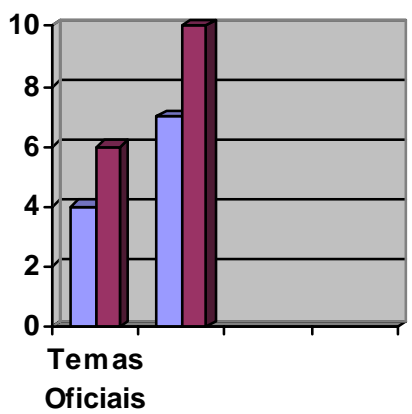

meio intrahospitalar, tanto nos temas oficiais quanto nos temas livres.

Oficiais 
Quadro 01 - Temas oficiais referentes à temática saúde da criança

\begin{tabular}{|l|l|}
\hline \multicolumn{1}{|c|}{ REFERÊNCIA } & \multicolumn{1}{c|}{ TíTULO } \\
\hline $\begin{array}{l}\text { Ano: } 1971 \\
\text { XXIII CBEn - Manaus }\end{array}$ & $\begin{array}{l}\text { "Participação da enfermagem na implementação do BCG } \\
\text { intradérmico no Brasil " } \\
\text { "Programa de investigação sobre a praticabilidade da vacinação } \\
\text { BCG intradérmica direta em crianças de escolas primárias" }\end{array}$ \\
\hline $\begin{array}{l}\text { Ano: } 1972 \\
\text { XXIV CBEn - Belo Horizonte }\end{array}$ & "Estudo da Monilíase no recém nascido" \\
\hline $\begin{array}{l}\text { Ano: } 1973 \\
\text { XXV CBEn - João Pessoa }\end{array}$ & $\begin{array}{l}\text { "Estudo e observação do comportamento de mães e gestantes } \\
\text { em relação aos cuidados que dispensam ou dispensarão a seus } \\
\text { filhos" } \\
\text { "Atividades discentes e docentes organizadas a partir das } \\
\text { funções da enfermeira de pediatria" }\end{array}$ \\
\hline $\begin{array}{l}\text { Ano: } 1974 \\
\text { XXVI CBEn - Curitiba }\end{array}$ & $\begin{array}{l}\text { "Evolução das temperaturas axilar e retal de recém-nascidos } \\
\text { normais, nas primeiras 48 horas de vida" }\end{array}$ \\
\hline $\begin{array}{l}\text { Ano: } 1975 \\
\text { XXVII CBEn - Salvador }\end{array}$ & $\begin{array}{l}\text { "Observação clínica das ocorrências no processo de } \\
\text { mumificação; queda do coto e cicatrização da ferida umbilical } \\
\text { em recém-nascidos submetidos a dois cuidados de higiene" } \\
\text { "Estudo do pH e densidade urinária na reidratação do lactante" } \\
\text { "Papel das enfermeiras junto as mães de crianças } \\
\text { hospitalizadas" }\end{array}$ \\
\hline $\begin{array}{l}\text { Ano: } 1976 \\
\text { XXVIII CBEn - Rio de Janeiro }\end{array}$ & "O estudo de candidíase oral em recém -nascido" \\
\hline
\end{tabular}

Quadro 02 - Temas livres referentes à temática saúde da criança

\begin{tabular}{|c|c|}
\hline REFERÊNCIA & TíTULO \\
\hline $\begin{array}{l}\text { Ano: } 1970 \\
\text { XXIII CBEn - São Paulo }\end{array}$ & $\begin{array}{l}\text { "Um método de colheita de excretas em Pediatria" } \\
\text { "Assistência Integral ao prematuro, possibilidade atual e futura" } \\
\text { "Cirurgia neo-natal sob o ponto de vista da enfermagem" }\end{array}$ \\
\hline $\begin{array}{l}\text { Ano: } 1972 \\
\text { XXIII CBEn - Belo Horizonte }\end{array}$ & "A enfermagem domiciliária no tratamento da diarréia infantil" \\
\hline $\begin{array}{l}\text { Ano: } 1975 \\
\text { XXIII CBEn - Salvador }\end{array}$ & "Assistência de enfermagem em berçário" \\
\hline $\begin{array}{l}\text { Ano: } 1976 \\
\text { XXIII CBEn - Rio de Janeiro }\end{array}$ & $\begin{array}{l}\text { "Recursos Humanos de enfermagem para assistência à saúde } \\
\text { inclusive materno infantil" } \\
\text { "Assistência respiratória em pós-operatório de cirurgia cardíaca } \\
\text { infantil" } \\
\text { "Uma experiência com consulta de enfermagem para criança" } \\
\text { "Controle domiciliar de prematuro no Hospital do Servidor } \\
\text { Público Estadual, no primeiro ano de vida - Resultados obtidos } \\
\text { em } 5 \text { anos de atividades" } \\
\text { "A atuação da enfermeira no subprograma - Assistência ao } \\
\text { menor- da Secretaria do Bem Estar Social da Prefeitura do } \\
\text { Município de São Paulo (SBES)" }\end{array}$ \\
\hline
\end{tabular}




\begin{tabular}{|l|l|}
\hline \multirow{1}{*}{ Ano: 1978} & "Importância da assistência de enfermagem, na realimentação \\
XXIII CBEn - Belém & "Prescrição de enfermagem uma experiência nas áreas de \\
& enfermagem médico - cirúrgico e pediátrica" \\
& "Participação da enfermagem no Programa Materno- Infantil da \\
& Secretaria de saúde do estado do Pará" \\
& "Diagnósticos mais freqüentes em pediatria e eficiência dos \\
Serviços de Saúde" & "Uma experiência no berçário no Hospital Ana Nery aplicando o \\
processo de enfermagem" \\
"Intoxicação Exógena em pediatria e a atuação de enfermagem" \\
"Avaliação comparativa de 3 procedimentos de collheita de urina \\
em lactantes para exames bacteriológicos"
\end{tabular}

\section{CONCLUSÕES}

Com base nos resultados, conclui-se que a temática - Saúde da Criança no recorte temporal de 1970 a 1979 foi pouco abordada, sendo que o XXVII CBEn realizado em Salvador em 1975 e o XXX CBEn realizado em Belém, em 1978 apresentaram os maiores percentuais, ou seja, 1,53\% de temas oficiais e $3,08 \%$ de temas livres relacionados à temática respectivamente.

No tocante da produção científica referente a temática Saúde da Criança, pode-se verificar um baixo percentual de trabalhos publicados nos Anais do CBEn nos anos 70.

No âmbito da temática - Saúde da Criançaconstata-se que o meio intrahospitalar ganhou destaque, pois na década de 70 predominava o modelo biomédico e a assistência à criança estava voltada para as ações curativas, visto que o hospital tinha uma posição central na prestação de saúde.

\section{REFERÊNCIAS BIBLIOGRÁFICAS}

BERTOLOZZI, M.R.; FONSECA, R.M.G.S; FORCELLA, H.T. Congressos Brasileiros de Enfermagem: Meio Século de Compromisso da ABEn. Brasília: ABEn, 2000.

BRIGNOLI, H.P.; CARDOSO, C.F.S. Os métodos da história. 3.ed. Rio de Janeiro: Graal, 1983.

CARDOSO, C.F.S. Uma Introdução à história, 10. ed. São Paulo: Brasiliense, 1994.

CARVALHO, A.C. de. Associação Brasileira de Enfermagem- 1926-1976: documentário. Brasília: Associação Brasileira de Enfermagem, 1976.

CERVO, A.L.; BERVIAN, P.A . Metodologia Científica. 4. Ed. São Paulo: Markron Books, 1996.

CEBEn - CONGRESSO BRASILEIRO DE ENFERMAGEM, 24, 1972, Belo Horizonte. Anais... Belo Horizonte: ABEn, 1972.

$$
\text { , 25, 1973, João Pessoa. }
$$

Anais... João Pessoa: ABEn, 1973.

Anais... Curitiba: ABEn, 1974.

$$
\text { 26, 1974, Curitiba. }
$$

27, 1975, Salvador.
28, 1976, Rio de Janeiro.

Anais... Rio de Janeiro: ABEn, 1976.

29, 1977, Santa Catarina.

Anais... Santa Catarina: ABEn, 1977.

30, 1978, Belém. Anais...

Belém: ABEn, 1978. 31, 1979, Ceará. Anais...

Ceará: ABEn, 1979.

FERRIANI, M.G.C; ROCHA, S.M.M; CANO, M.A.T. A prática da enfermagem pediátrica no Estado de São Paulo. In: Congresso Brasileiro de Enfermagem, 37, 1985, Recife. Anais... Recife: ABEn, 1985. p.371-381.

OLIVEIRA, I.C.S. A Institucionalização da Enfermagem Pediátrica: da Prática à Especialidade nos anos 70. Projeto integrado de pesquisa /CNPq. Brasília. 2003.

REBEn - REVISTA BRASILEIRA DE ENFERMAGEM, Rio de Janeiro, v.23: 1970.

v.24: 1971. , Rio de Janeiro,

Texto recebido em 13/04/2006

Publicação aprovada em 30/04/2006 